\title{
Erythropoietic Protoporphyria. A Major Skin Involvement
}

\author{
Tishler PV* \\ Channing Division of Network Medicine, Brigham and Womens Hospital and Harvard \\ Medical School, USA
}

*Corresponding author: Peter V Tishler, MD, Channing Division of Network Medicine, 181 Longwood Avenue, Boston Massachusetts 02115, USA, Tel: 617-5252123; E-mail: peter.tishler@channing.Harvard.edu

\section{Abstract}

Erythropoietic Protoporphyria (EPP) is a relatively rare disease [1], probably less so than the rare acute intermittent porphyria. Virtually all patients with EPP are symptomatic as young children. For example, a 10-year old boy was referred to me, a porphyria specialist, because of cutaneous photosensitivity since infancy. With sun exposure, his skin quickly developed tingling and subsequently swelling, petechiae, burning, stinging, erythema and pain, and rarely potential lethal liver dysfunction. In general, affected patients, including this boy, avoid being outside or with the sun. When outside, they often wear total-body clothes to avoid body exposure to sun and/or heat. Ultimately, they lead, without treatment, to scarring and resorption of the nose, auricles and fingers. The few physicians who specialize in the porphyrias do encounter them relatively frequently. I have seen many youngsters and adults with this syndrome, and have advised and treated them regarding probably preventive EPP therapy.

Keywords: Erythropoietic Protoporphyria; Porphyria; Protoporphyrin

\section{Introduction}

I have seen many individuals, youngsters and adults, with appropriate physical manifestations but no diagnosis of any porphyria. I instruct them to bring to me a 24 -hour urine and spot stool, to be sent to the highly specialized and diagnostic Porphyria Laboratory in Galveston Texas [2], to be analyzed for the acute porphyrias (acute intermittent porphyria, coproporphyria, variegate porphyria). These analyses are usually not abnormal and diagnostic. At the same time, we obtain and send to the Porphyria Laboratory an anticoagulated blood sample for the analysis of free protoporphyrin, the source of EPP. In EPP, for which the synthetic biochemistry of heme synthesis is in Figure 1, studies are usually virtually normal except for an increase in erythrocyte free protoporphyrin (normal 20-80 ug/dl; in EPP, almost always $>500 \mathrm{ug} / \mathrm{dl}$ ), and occasionally in urinary deltaaminolevulinic acid (ALA; normal 0-7ug/24 hr.; considerably higher in one type of EPP). Thus, there are two types of EPP. Although both types do have very elevated serum free protoporphyrin concentrations, in urine one is without increased ALA (type 1) and the other has very increased ALA (type 2). Type 1 EPP is almost always an autosomal recessive syndrome, resulting from inheritance of mutations in the two ferrochelatase allele genes that control the synthesis of protoporphyrin IX to hemoglobin (Figure 1), but rarely an unusual single ferrochelatase gene deficiency that causes EPP. The type 2 EPP syndrome with an elevated ALA is a less frequent Xlinked syndrome resulting from a gene mutation in ALA synthetase (Figure 1, ALA synthesis). Genotype analysis, by sending anticoagulated blood to the genotypic laboratory in New York City [3], can be subjected to virtually all of these patients, with informative results. 


\section{Clinical Dermatology Open Access Journal}

Investigating the family members of this EPP patient for gene deficiencies or the same syndrome is indicated.

The current treatment of EPP is potentially excellent. Previously, over a number of years, therapies with betacarotene, N-acetyl-L-cysteine or vitamin $\mathrm{C}$ are of little or no benefit. Treatment with oral colestipol, which may absorb pathologic materials that are secreted into the intestine, is active in both a relatively small study [4] and in children currently using it properly. A newer and superior medication, afamelanotide [5], has been developed and used superbly in Europe. It has not been approved by the U. S. Food and Drug Administration yet for use in this country, and will eventually be used and therapeutic. In addition to proper treatment, the EPP therapy must also reduce the amount and concentration of protoporphyrin within the body, since this is probably generating and involved in EPP problems.

The excessive protoporphyrin is secreted into the biliary tract, leading occasionally to biliary tract obstruction and/or gall bladder stones and rarely to hepatic dysfunction. A small percentage of affected children develop potentially lethal hepatic failure, for which treatment is hepatic transplantation. Reducing the protoporphyrin in the colon with therapy should be investigated. Both colestipol and afamelanotide, major therapeutic agents, have not been evaluated for this, but should complete the analyses soon.

For physicians with expertise in EPP or those who read and understand the aforementioned description of EPP and its therapy when the patient's EPP diagnosis is likely, all physicians can make the diagnosis of and treat EPP. Physicians who are under-educated in EPP should, if necessary, consult individuals at the American Porphyria Foundation [6], asking for appropriate education regarding EPP or referral of the patient to a porphyria specialist. The number of nationwide porphyria specialist physicians is small, but members of the American Porphyria Foundation can, if necessary, refer the patient to a porphyria specialist.

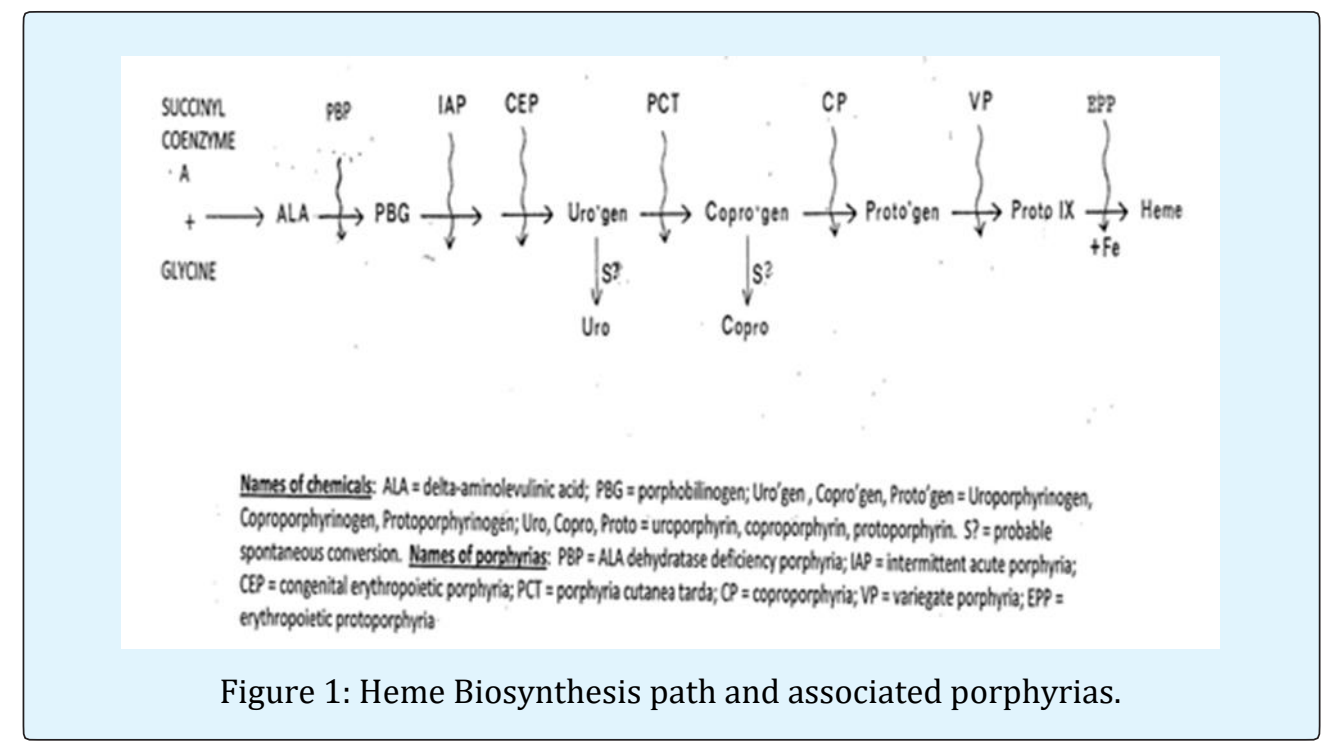

\section{References}

1. Tishler PV (2016) Porphyrias: Diagnosis and Management; In: Abutalib SA (Eds.) Non-malignant Hematology, Springer International Publishing, Switzerland, pp: 23-28.

2. The Porphyria Laboratory, University of Texas Medical Branch, 700 Harborside Drive, Eving Hall 3.102, Galveston, Texas 77555-1109.

3. Mount Sinai Genetic Testing Laboratory, Porphyria DNA Testing.
4. Tishler PV, Rosner B (2014) Treatment of ErythropoieticProtoporphyria with the Oral Sorbent Colestipol: A Proof-of-Concept Clinical Trial. J AmerAcad Dermatol 70(2): 391-392.

5. Langendonk JG, Balwani $\mathrm{M}$, Anderson KE, Bonkovsky HL, Anstey AV, et al. (2015) Afamelanotide for Erythropoietic Protoporphyria. New Eng J Med 373: 48-59.

6. American Porphyria Foundation. 